\title{
Clinical Infectious Diseases
}

\section{Reply to author}

--Manuscript Draft--

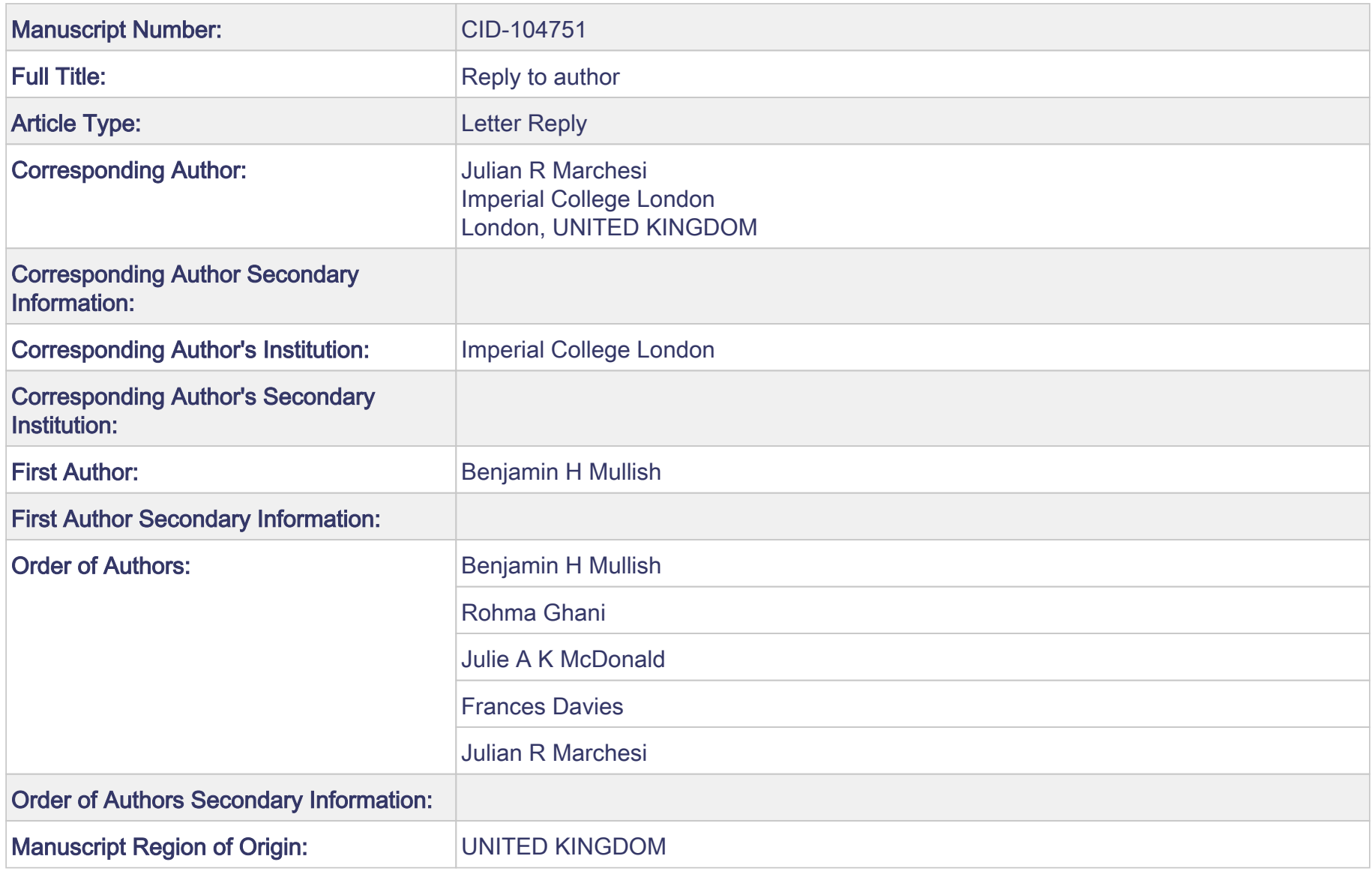


Click here to access/download;Cover Letter;cover letter CID response 25092020.pdf

\section{Imperial College London}

Division of Digestive Disease

Department of Metabolism, Digestion and Reproduction

Faculty of Medicine, Imperial College London

St Mary's Hospital Campus

$10^{\text {th }}$ Floor, QEQM Wing

South Wharf Road, Paddington

London, W2 1NY, United Kingdom

Tel: +44 (0)2033126454 Fax: +44 (0)2077249369

i.marchesi@imperial.ac.uk

https://www.imperial.ac.uk/people//.marches

Professor Julian R Marchesi PhD

Professor of Digestive Health

$25^{\text {th }}$ Sept 2020

Dear Prof. Schooley,

We wish to submit our response to Woodworth and colleagues' letter in CID.

Yours sincerely,

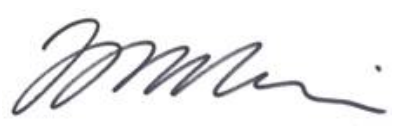

Professor Julian Marchesi

Professor of Digestive Health 
1 Letter

2 Reply to author

3

4 Benjamin H Mullish ${ }^{1,2 \ddagger}$, Rohma Ghani ${ }^{1,3 \ddagger}$, Julie A K McDonald ${ }^{4}$, Frances Davies ${ }^{3}$, Julian R Marchesi*1,5

5

6 1. Division of Digestive Diseases, Department of Metabolism, Digestion and Reproduction, Imperial $7 \quad$ College London, London, UK.

8 2. Departments of Gastroenterology and Hepatology, St Mary's Hospital, Imperial College $9 \quad$ Healthcare NHS Trust, London, UK.

10 3. Department of Infection, Imperial College Healthcare NHS Trust, London, UK.

4. MRC Centre for Molecular Bacteriology and Infection, Imperial College London, London, UK.

5. School of Biosciences, Cardiff University, Cardiff, UK.

13

${ }^{\ddagger}$ Contributed equally to this manuscript.

Professor Julian R Marchesi

Division of Digestive Diseases South Wharf Road Paddington, London

W2 1NY 
26

27 Word count:

477

28

29 Key words: antimicrobial resistance; gut microbiome; fecal microbiota transplant; multi-drug 30 resistant bacteria; allogeneic hematopoietic cell transplantation

31 
We thank Woodworth and colleagues for their letter [1] about our manuscript [2], and are happy to provide clarification.

Our study described outcomes after fecal microbiota transplantation (FMT) in patients with intestinal colonization with multi-drug resistant organisms (MDROs), who were either at risk of invasive MDRO disease (principally patients with hematological malignancy) or those with prior recurrent invasive MDRO disease (including patients with recurrent urinary tract infections (UTIs)). As described in the study, $41 \%$ of patients $(n=7 / 17)$ underwent intestinal decolonization (as assessed by culture of rectal swabs/ stool samples) over the course of study follow-up, a figure comparable to a reported spontaneous decolonization rate of $48 \%$ [3]. As the correspondents have themselves noted [4], prior FMT studies for attempted decolonization of intestinal MDROs have assessed decolonization endpoints in a relatively consistent fashion, with culture and PCR being the predominant laboratory tools used, and a binary 'present/ not present' being the typical reporting outcome. Current best clinical practice in this area employs a similar approach. In this study, we did not assess titres of specific MDROs or quantify levels of antibiotic resistance genes (ARGs) within stool. Interestingly, in the context of FMT for recurrent $C$. difficile infection (rCDI), a post-FMT reduction in ARGs was more common in patients whose diarrhea resolved with FMT compared to non-responders [5]; evidently, similar assessment of this in the context of FMT for MDRO is of importance. It is also feasible, but unknown, as to whether, for instance, an FMT-related log-fold reduction in intestinal MDRO titer may reduce the possibly of translocation and bacteremia, even without true undetectability on a PCR assay. We agree that many uncertainties remain in relation to optimal FMT administration in non-CDI settings [6]. In our study, all patients received a single FMT only, apart from one patient who 
received a second FMT. This was a patient with recurrent UTIs; following the initial FMT, the frequency of their UTIs reduced, although they still did develop at least one symptomatic infection (albeit with an organism sensitive to oral antibiotics). As such, the patient underwent a second FMT at six months after the initial one; this was successful, with no further UTIs occurring over the course of study follow-up.

Despite the modest decolonization rate that we observed, our major novel finding was of clinical benefits in FMT recipients, including reduced antibiotic duration, bacteremia and length of inpatient stay compared to a matched group not receiving FMT [2]. Our data build upon those in the FMT/ rCDI literature, whereby reduction in bacteremia and length of stay - together with improved survival - was observed in FMT-treated patients compared to those administered antibiotics alone [7]. We agree that a priority for future studies is evaluation of mechanisms by which FMT exerts these effects, with restoration of altered gut barrier integrity and modulation of systemic immunity being two areas of key interest [8-10].

Funding: The Department of Metabolism, Digestion and Reproduction at Imperial College London receives funding from the National Institute of Health Research (NIHR) Biomedical Research Centre (BRC) based at Imperial College London and Imperial College Healthcare NHS Trust. BHM is the recipient of an NIHR Academic Clinical Lectureship. JAKM is the recipient of a Wellcome Trust Institutional Strategic Support Fund (ISSF) fellowship. FD receives funding from the Medical Research Council (MRC) Clinical Academic Research Partnership Scheme.

Acknowledgements: Nil. 
80 Potential conflicts of interest: BHM reports personal fees from Finch Therapeutics Group, outside

81 the submitted work. JRM reports personal fees from EnteroBiotix Ltd, outside the submitted work.

82 
1. Woodworth MH, Kwon JH, Kraft CS. An Ounce of Prevention is Equivalent to How Much Decolonization Exactly? Clin Infect Dis 2020;

2. Ghani R, Mullish BH, McDonald JAK, et al. Disease Prevention Not Decolonization: A Model for Fecal Microbiota Transplantation in Patients Colonized With Multidrug-resistant Organisms. Clin Infect Dis 2020; Available at: https://academic.oup.com/cid/advancearticle/doi/10.1093/cid/ciaa948/5873448. Accessed 23 September 2020.

3. Davido B, Moussiegt A, Dinh A, et al. Germs of thrones - spontaneous decolonization of Carbapenem-Resistant Enterobacteriaceae (CRE) and Vancomycin-Resistant Enterococci (VRE) in Western Europe: Is this myth or reality? Antimicrob Resist Infect Control 2018; 7.

4. Woodworth MH, Hayden MK, Young VB, Kwon JH. The Role of Fecal Microbiota Transplantation in Reducing Intestinal Colonization with Antibiotic-Resistant Organisms: The Current Landscape and Future Directions. Open Forum Infect Dis 2019; 6:1-9.

5. Millan B, Park H, Hotte N, et al. Fecal Microbial Transplants Reduce Antibiotic-resistant Genes in Patients with Recurrent Clostridium difficile Infection. Clin Infect Dis 2016; 62:1479-1486. Available at: http://www.ncbi.nlm.nih.gov/pubmed/27025836. Accessed 5 October 2019.

6. Allegretti JR, Mullish BH, Kelly C, Fischer M. The evolution of the use of faecal microbiota transplantation and emerging therapeutic indications. Lancet 2019; 394:420-431. Available at: https://www.thelancet.com/journals/lancet/article/PIIS0140-6736(19)31266-8/fulltext\#. Accessed 5 August 2019.

7. laniro G, Murri R, Sciumè GD, et al. Incidence of bloodstream infections, length of hospital stay, and survival in patients with recurrent clostridioides difficile infection treated with fecal microbiota transplantation or antibiotics a prospective cohort study. Ann Intern Med 2019; 

December 2019.

108

8. van Lier YF, Davids M, Haverkate NJE, et al. Donor fecal microbiota transplantation ameliorates intestinal graft-versus-host disease in allogeneic hematopoietic cell transplant recipients. Sci Transl Med 2020; 12:8926. Available at: http://stm.sciencemag.org/. Accessed 24 September 2020.

9. Kim SM, DeFazio JR, Hyoju SK, et al. Fecal microbiota transplant rescues mice from human pathogen mediated sepsis by restoring systemic immunity. Nat Commun 2020; 11:23542354.

10. Burrello C, Garavaglia F, Cribiù FM, et al. Therapeutic faecal microbiota transplantation controls intestinal inflammation through IL10 secretion by immune cells. Nat Commun 2018; 9:5184. Available at: http://www.nature.com/articles/s41467-018-07359-8. Accessed 23 February 2019. 